\title{
Tunable charge donation and spin polarization of metal adsorbates on graphene using applied electric field
}

\author{
Jae-Hyeon Parq, ${ }^{1}$ Jaejun Yu, ${ }^{1}$ Young-Kyun Kwon, ${ }^{2}$ and Gunn $\mathrm{Kim}^{2}$, * \\ ${ }^{1}$ Center for Strongly Correlated Materials Research, \\ Department of Physics and Astronomy, Seoul National University, Seoul 151-747, Korea \\ ${ }^{2}$ Department of Physics and Research Institute for Basic Sciences, Kyung Hee University, Seoul 130-701, Korea
}

(Dated: August 30, 2021)

\begin{abstract}
Metal atoms on graphene, when ionized, can act as a point charge impurity to probe a charge response of graphene with the Dirac cone band structure. To understand the microscopic physics of the metal-atom-induced charge and spin polarization in graphene, we present scanning tunneling spectroscopy (STS) simulations based on density functional theory calculations. We find that a Cs atom on graphene are fully ionized with a significant band bending feature in the STS, whereas the charge and magnetic states of $\mathrm{Ba}$ and La atoms on graphene appear to be complicated due to orbital hybridization and Coulomb interaction. By applying an external electric field, we observe changes in charge donations and spin magnetic moments of the metal adsorbates on graphene.
\end{abstract}

PACS numbers: 73.22.-f, 68.43.Bc, 73.20.Hb

Because of its Dirac cone structure in the energy dispersion, electrons in graphene behave like two-dimensional (2D) massless Dirac fermions at low energies [1]. The presence of the Dirac cone structure near the Fermi level plays a vital role in the determination of physical properties of pristine graphene. In practical applications, however, the influences of adatoms and the substrate should also be considered. An adsorbed metal atom may alter the physical properties, such as the chemical potential and the effective dielectric constant, of graphene through charge transfer and orbital hybridization on graphene 22 5. Recently, a model based on an assumption of weak coupling between graphene and adatom was suggested to study 2D quantum-relativistic phenomena on graphene with a charged metal atom [6. This model study predicted that an atomic collapse-like condensed matter phenomenon may arise [6].

Although several theoretical models have been suggested for charge impurities in graphene, there has been no attempt to describe a realistic model with ionized atoms on graphene including the effects of charge transfer, orbital hybridization, and Coulomb screening simultaneously. Since the amount of charge transfer depends sensitively on coverage [7, which may also can affect adsorption configurations and bonding characters [8, it is necessary to take a sufficiently large cell for simulations at low coverage.

In this Letter, we present the scanning tunneling spectroscopy (STS) simulations of graphene with metal adatoms, based on density functional theory (DFT) calculations. Our STS simulations were performed using a large-sized supercell for the low coverage of metal adsorbates on graphene, and revealed the effects of charge transfer, orbital hybridization, and Coulomb screening between graphene and metal adatoms. We selected three metal atoms, cesium $(\mathrm{Cs})$, barium (Ba), and lanthanum (La), to investigate the dependence on the electron affinity of adatoms. We observed band bending near the adatom by monitoring our simulation data through the van Hove singularity (vHS) lines in the STS plot. Cs atoms were fully ionized on graphene with a significant band bending feature in the STS, whereas $\mathrm{Ba}$ and La atoms exhibited complicated electronic and magnetic properties due to orbital hybridization and Coulomb interactions with graphene. We also studied the effects of an applied external electric field (E-field) on charge donations and magnetic moments of adatoms on graphene.

We performed first-principles DFT calculations, using the OpenMX code 9 , within the local spin density approximation (LSDA) and the generalized gradient approximation (GGA) 10. General features of the computational results were almost identical for the two approximations. All figures presented in this Letter were obtained from the results of GGA calculations. The OpenMX code employs a linear combination of pseudo-atomic orbitals (LCPAO) [11. We employed Troullier-Martins-type norm-conserving pseudopotentials [12, 13] in a factorized separable form with partial core correction [14. Real-space grid techniques [15] were used in numerical integration with energy cutoff up to 200 Ry for LSDA and 300 Ry for GGA. In our calculations, $8 \times 8 \times 1 k$-points were sampled for calculations with a small unit cell containing 60 carbon atoms, and $2 \times 2 \times 1 k$-points for calculations with a large unit cell of 800 carbon atoms. The structures were relaxed until the Hellman-Feynman force became smaller than $6 \times 10^{-4}$ Hartree $/ a_{\mathrm{B}}$, where $a_{\mathrm{B}}$ is the Bohr radius.

To determine the adsorption site of metal adatoms, we used a simple orthorhombic unit cell consisting of 60 carbon atoms and one metal atom, as shown in Fig. 1(a). Among three possible candidate sites (on-top, bridge, and hollow sites), the hollow site was found to be preferable for all three metal atoms, which agrees with the previous work [16] on the Cs atom where the nature of binding was attributed to ionic bonding [2, 16 [18]. The calcaulated adsorption 

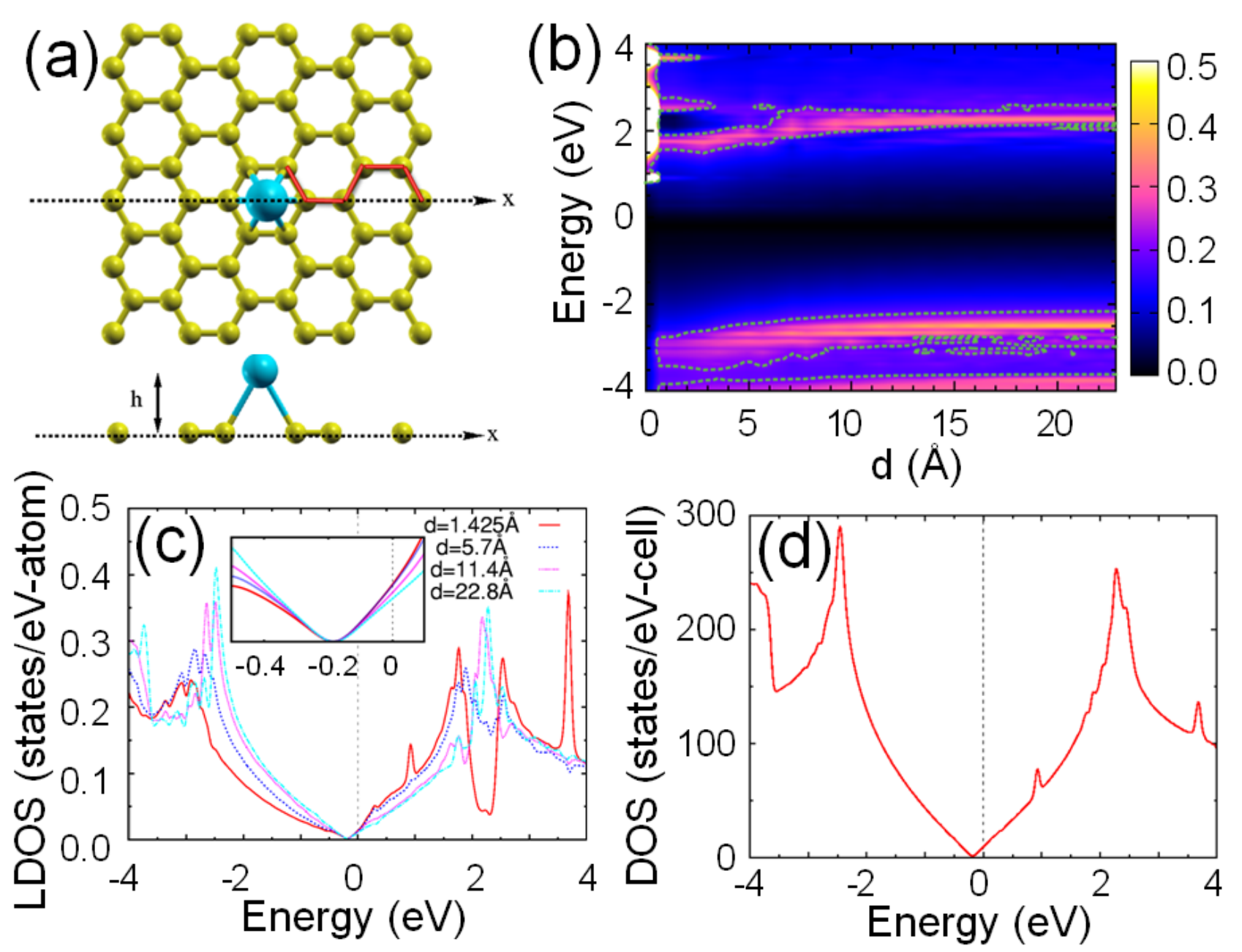

FIG. 1: STS simulations for Cs-adsorbed graphene. (a) Small unit cell model. (b) 2D simulated STS map obtained using the large unit cell. $d$ denotes the $x$-components of the amchair line depicted by the red line in (a). (c) LDOSs at distances of $d=$ $1.425,5.7,11.4$, and $22.8 \AA$ correspond to vertical cuts of the STS map. Inset shows the magnified LDOS near the Dirac point. (d) Total DOS of Cs-adsorbed graphene. The Fermi level is set to zero.

heights exhibit a trend related to the ionic radius of the metal atom [2].

Next, we examined charge transfer and magnetic exchange interaction between the adatom and the graphene layer. The calculated charge and magnetic moment for each metal atom are listed in Table I] as obtained by the Voronoi charge analysis [19]. To ensure the proper description of the charge response in the graphene layer, we introduced a large unit cell consisting of approximately 800 carbon atoms and a metal atom. All the results presented hereafter were obtained from the large cell calculations.

According to our calculations, a Cs atom donates almost one electron to graphene to be a $\mathrm{Cs}^{+}$ion in agreement

TABLE I: The position (height), charge transfer, and atomic magnetic moment of each metal atom adsorbed on large unit-cell graphene, and total magnetic moment of each model system. GGA results are shown in the Voronoi charge scheme.

\begin{tabular}{cccr}
\hline \hline & Cs & Ba & La \\
\hline Height $(\AA)$ & 3.01 & 2.65 & 2.38 \\
Charge $(e)$ & 0.95 & 1.20 & 1.27 \\
Atomic moment $\left(\mu_{\mathrm{B}}\right)$ & 0.00 & 0.40 & 1.27 \\
Total moment $\left(\mu_{\mathrm{B}}\right)$ & 0.00 & 0.47 & 1.86 \\
\hline \hline
\end{tabular}



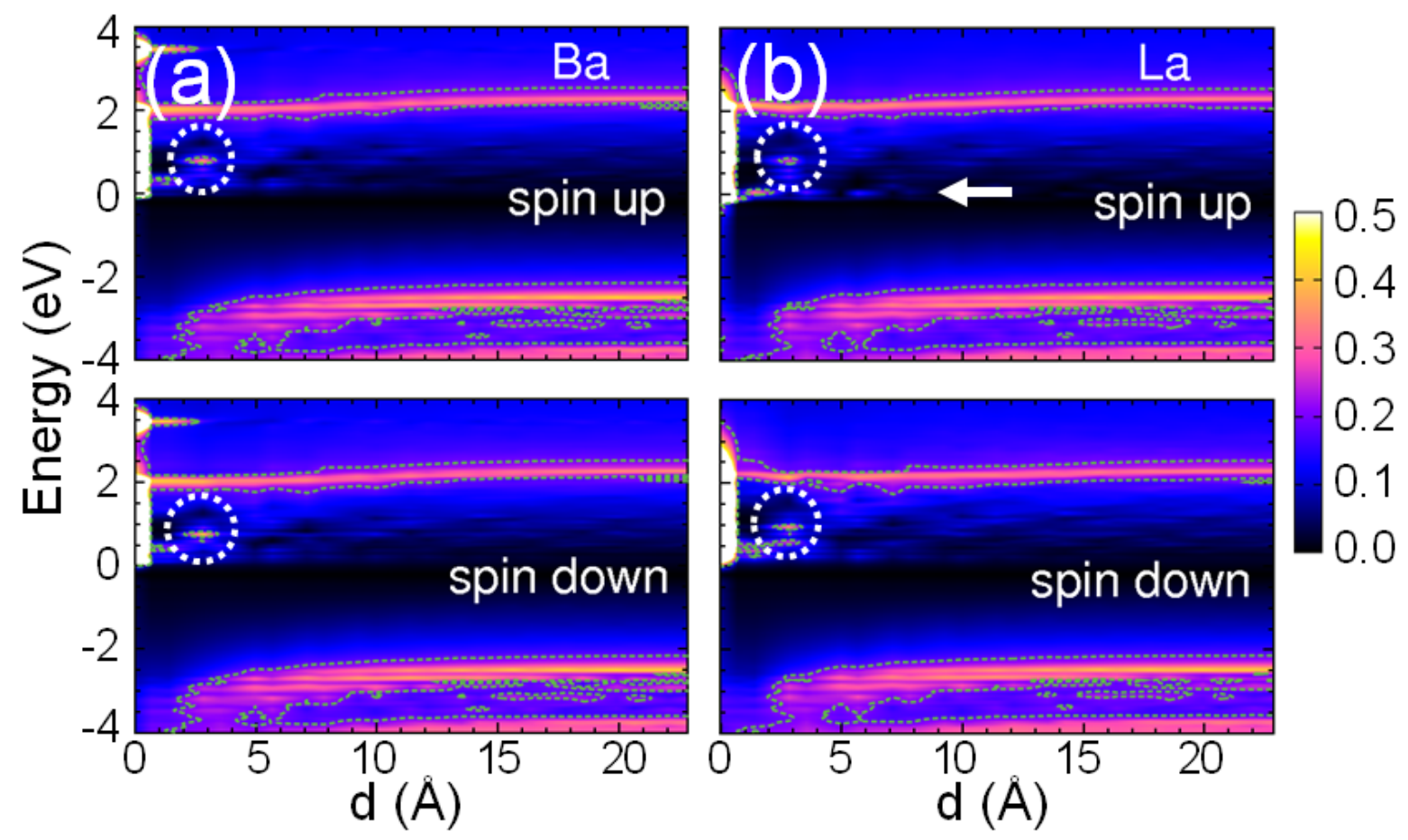

FIG. 2: Simulated STS maps of (a) Ba- and (b) La-adsorbed graphene layers. The arrow in (b) indicates the location of the state corresponding to Fig. 5(a). The dashed-lined circles denote symptoms of the covalent characters.

with an XPS measurement 20. For a Ba or a La atom on graphene, on the other hand, it turned out that the amount of transferred electrons is $1.2-1.3 \mathrm{e}$. It means that such an adsorbed atom is not fully ionized as observed in previous reports [2, 7]. Since the electron affinity of graphene is not very high, Ba or La may not exceed the critical potential strength [6] on graphene in the absence of external E-field. Besides, strong orbital hybridization between graphene states and $d$ electrons of $\mathrm{Ba}$ or La prevents the metal atoms from being fully ionized. This finding implies that neither Ba nor La acts as a supercritical impurity for an atomic collapse-like phenomenon [6], in contrast to an ealier study proposing that divalent and trivalent dopants, such as alkaline-earth and rare-earth metals by used for an experimental investigation of the phenomenon [6].

To simulate the STS data reflecting adatom bonding characters, we calculated the spatially-resolved local density of states (LDOS). Figures 1(b), 2(a), and 2(b) present 2D simulated STS maps obtained from the calculated LDOS of carbon atoms, along an armchair line, depicted in Fig. 1(a), from the impurity center to a distant region, the same way used in a previous study on a carbon nanotube (CNT) 21. The Fermi level was shifted upward by $\sim 0.2 \mathrm{eV}$ due to charge donation from the metal adsorbate to the graphene. In the conduction bands, the peak lines corresponding to the vHSs of the Cs-adsorbed graphene form a curve much more prominent than those of the Ba- and La-adsorbed cases. (for clarity, see isovalue lines). On the other hand, the apparent band bending phenomenon in the valence bands are more clearly shown in Ba- and La-adsorbed graphene layers than in Cs-doped layer. The differences between band bending in the Cs-adsorbed case and those in $\mathrm{Ba}$ and La cases originate from the effects of orbital hybridization and the Coulomb potential. The slope of the asymmetric LDOS around the Dirac point remains intact independent of charge doping, as shown in Fig. 1(c). Interestingly, the total DOS [Fig. 1(d)] is almost symmetrical around the Dirac 


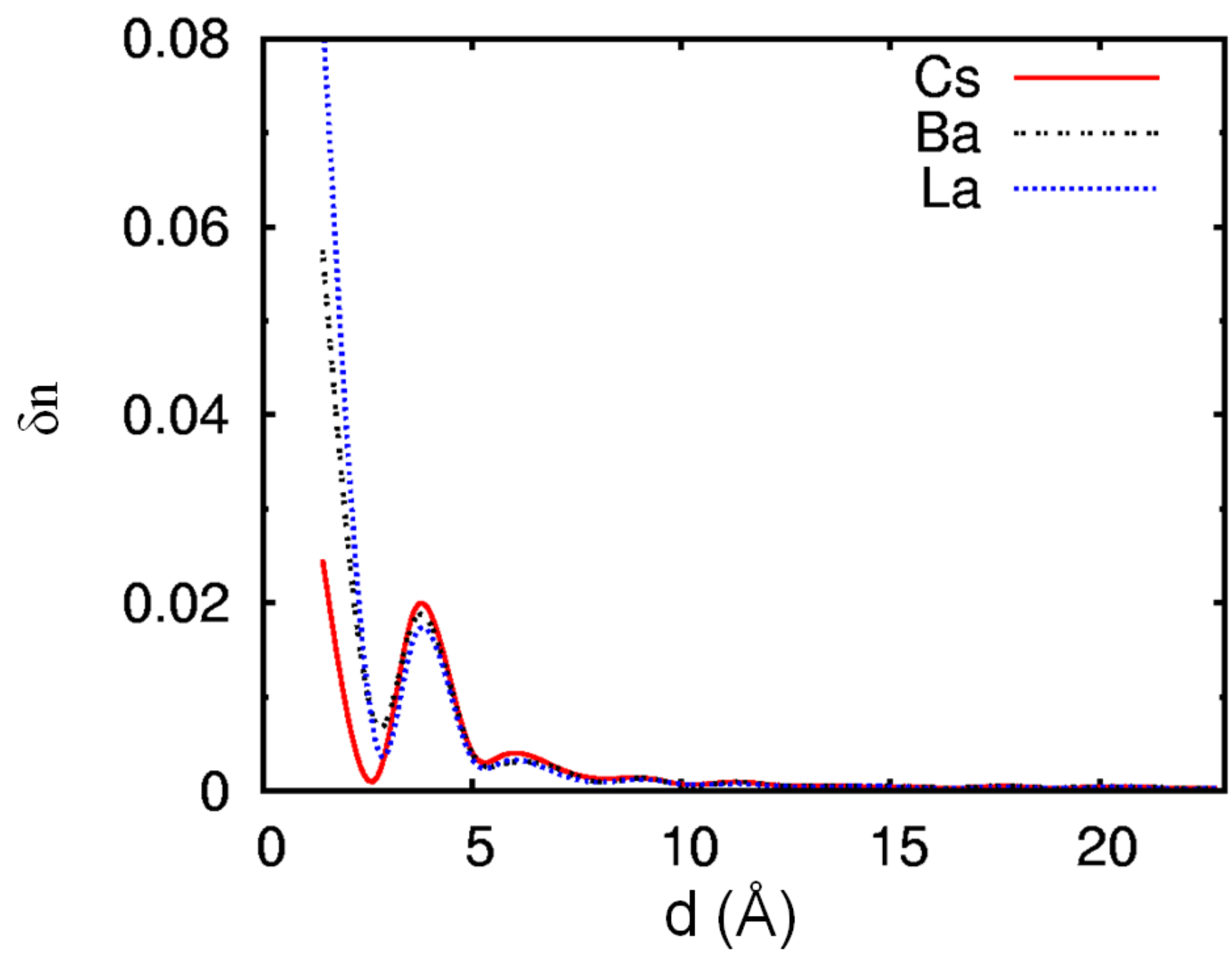

FIG. 3: The Friedel oscillation patterns caused by the charge impurities for Cs-, Ba- and La-adsorbed graphene layers. Within a few angstrom, the amplitudes of the charge density oscillaltions rapidly decrease for all three metal adsorbates.

point, which implies that distant graphene states compensate for the asymmetry near the impurity atom. Similar to a previous prediction by a tight-binding approach 22, our calculations show that the Dirac point is robust. The robustness of the Dirac point is still valid for the cases of Ba and La, even though there are strong spin polarization and orbital hybridization effects.

As summarized in Table I, spin magnetic moments on graphene, due to Ba and La atoms, were 0.47 and $1.86 \mu_{\mathrm{B}}$, respectively. Since the $\mathrm{Ba}$ and La atoms are not fully ionized on graphene, the magnetic moments, produced by the remaining electrons, influence the STS maps, as shown in Fig. 2. The $6 s$ orbitals of the Ba and La atoms on graphene are overlapped to the $5 d$ orbitals, and they are partially occupied. In addition, the relative positions of the up-spin and down-spin states in the $5 d$ orbitals around the Fermi level bring about the spin magnetic moments. It is clearly shown that the unoccupied $5 d$ orbitals of $\mathrm{Ba}$ and La are strongly hybridized with graphene states in a wide energy window. The partially occupied atomic states of Ba and La, especially for up-spin, are located right below the Fermi level, near the Dirac point. Furthermore, the STS maps reveals their covalent bonding characters, which are encircled in Fig. 2. In a previous CNT experiment, such a covalent bonding character between Ba and carbon $p$ orbitals was observed [23. For the Cs-adsorbed graphene, in contrast, no deformation was found in the Dirac point, since Cs is completely ionized and thus no partially occupied state exists. Moreover, Fig. 1(a), the STS map for the Cs case, does not display the covalent character spot as expected for the ionic bonding of Cs with $\mathrm{C}$ in graphene.

Ripples of brighter colors are displayed in our STS maps, as indicated by an arrow in Fig. 2(b). Such ripples are the consequence of strong orbital hybridization between adatoms ( $\mathrm{Ba}$ and $\mathrm{La}$ ) and graphene. Most of the ripples are present in the conduction bands; however, the up-spin valence band for La contains a decaying pattern of $d-p$ 


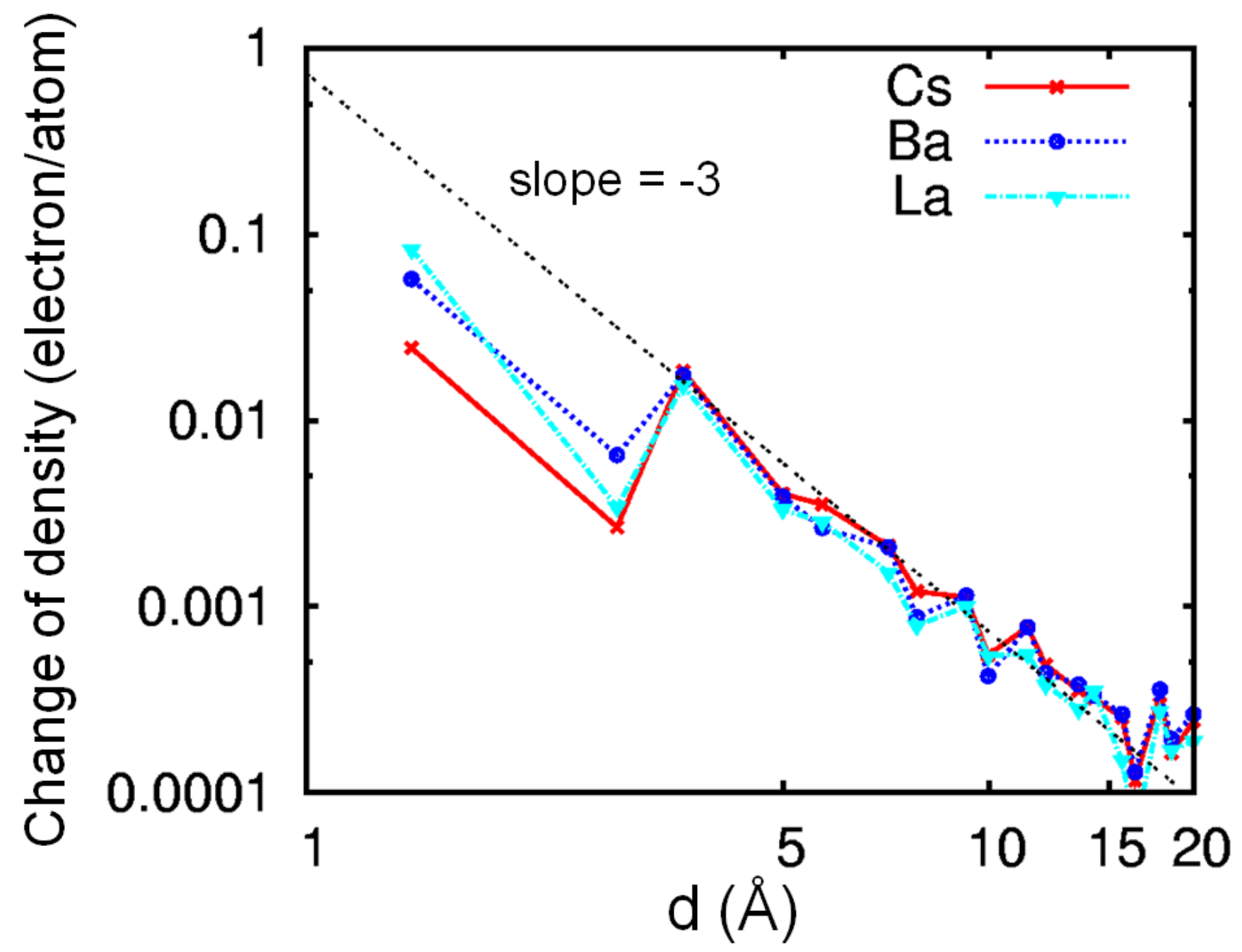

FIG. 4: Changes in electronic density of the graphene layer during metal atom adsorption. The infinite distance limit ( $0.0002 \mathrm{e}$ ) was already subtracted. Both axes are plotted on the logarithmic scale. The data were obtained from Voronoi charge analysis.

orbital hybridization. For Ba, on the other hand, a relatively weak decaying pattern appears in the up-spin valence band, since $5 d$ population in Ba is much smaller than in La. In contrast, the Cs-adsorbed graphene exhibits very weak orbital hybridization resulting in no ripples as seen in Fig. 1(a). From charge density analysis, we also found the Friedel oscillation patterns caused by the charge impurities on graphene (Fig. 3). Our results demonstrate that within $\sim 5 \AA$ from the metal adatom, the amplitudes of the charge density oscillaltions rapidly decrease for all three metal cases. Besides, asymptotically at large distances from the adatom, the induced charge density by the impurity charge is inversely proportional to the cube of the distance from the impurity [22, 24]. Our ab initio calculations, albeit the finite unit cell size, also show similar asymptotic behaviors (Fig. 4). Therefore, it has been confirmed that the effects of Coulomb interaction in metal-atom-adsorbed graphene are insensitive to orbital hybridization.

The difference between the behaviors of the $\mathrm{Cs}$ atom and those of $\mathrm{Ba}$ and $\mathrm{La}$ atoms on graphene is due to the relative positions and widths of energy levels between the $s$ and $d$ orbitals in the metal atoms. Figures 5 (a) and 5 (b) are schematic diagrams of the LDOSs of Cs and La, respectively, and obviously show this interesting aspect. Due to charge donation to graphene, the $6 \mathrm{~s}$ orbital of Cs becomes completely empty, and is separate from the $5 d$ orbitals with a broad width [Fig. 5(a)]. Different from this feature of Cs, the $6 \mathrm{~s}$ orbitals of the Ba and La atoms on graphene are overlapped to the $5 d$ orbitals, and they are partially occupied. In addition, the relative positions of the up-spin and down-spin states in the $5 d$ orbitals around the Fermi level bring about the spin magnetic moments, as seen in Fig. 5(b). Figure 5(c) shows DFT results of the local electronic structure in graphene near the La adsorbate. In Fig. 5(d), a charge density near the Fermi level shows a combination of $5 d$ orbitals of La with four lobes of adatoms and $p$ orbitals of carbon, and corresponds to a rippled pattern in Fig. 2(b). On the other hand, Fig. 5(e) reveals that 


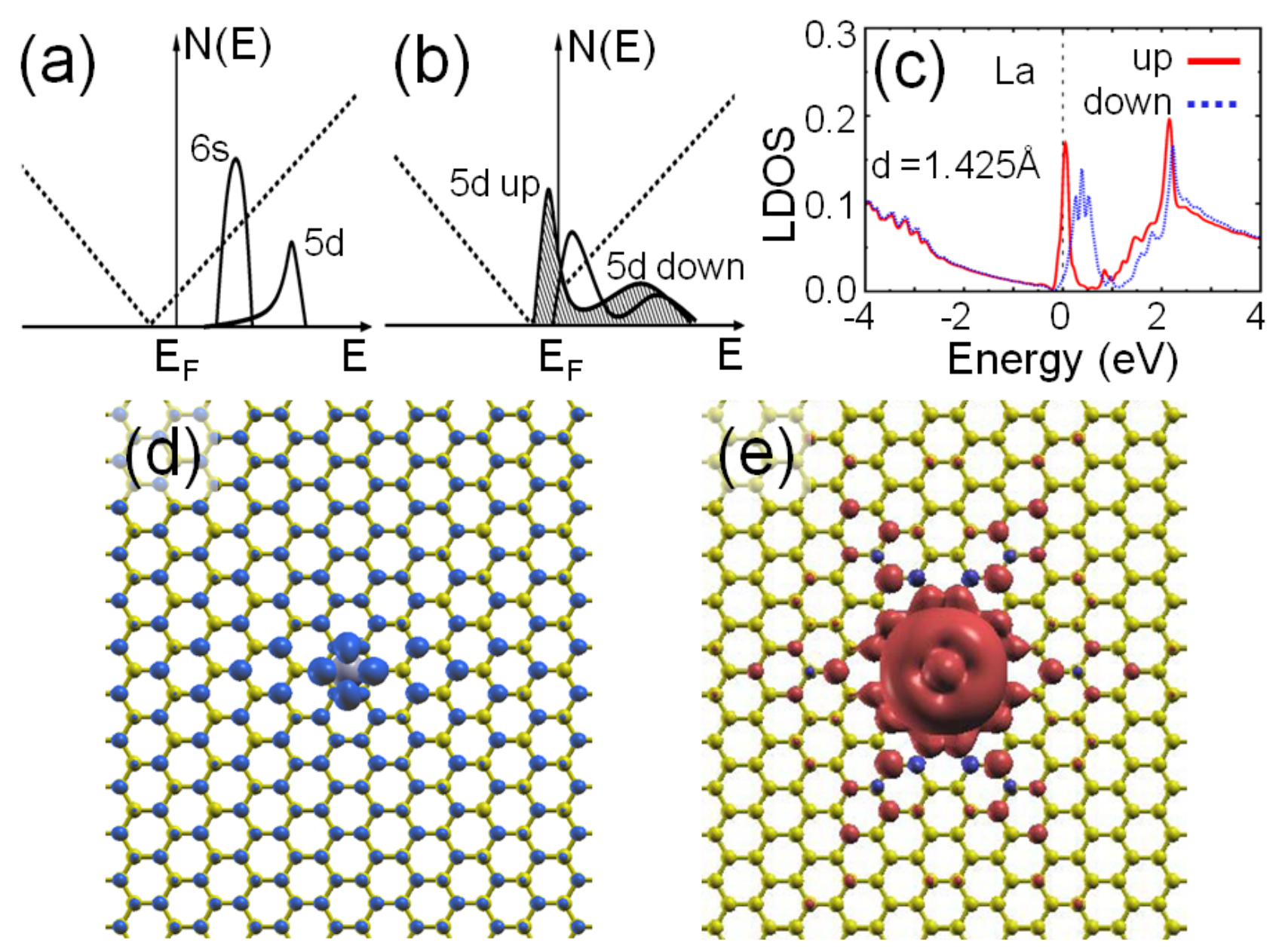

FIG. 5: Schematic energy diagrams of DOS of (a) Cs and (b) La on graphene. (c) DFT results for the La case at a distance of $d=1.425 \AA$. Isosurface plots of $(\mathrm{d})$ charge density associated with a majority spin eigenstate near the Dirac point at $E=-0.18 \mathrm{eV}$ from the Fermi level (Isovalue $=0.0001 \mathrm{e} / \AA^{3}$ ) and (e) the total spin density (Isovalues $= \pm 0.0001 e / \AA^{3}$ ) in the La-adsorbed graphene. In (e), the red and blue colors represent positive and negative values, respectively.

the magnetic moment of the system is mainly from the atomic spin moment of the metal atom on graphene, and the magnetic influence of the adatom on graphene seems negligible.

Finally, to explore how the ionic charge and magnetic moment of the metal adatom would change in the presence of external E-field, we applied external E-field to the La-adsorbed graphene. Using the gate bias voltage or local E-field from a scanning tunneling microscope tip, this study can be done in a real measurement. As shown in Table III, the La atom moves closer to the graphene sheet, and donates more electrons. Its ionic charge is, however, still smaller than $+2 e$ under the applied E-field of up to $2 \mathrm{~V} / \mathrm{nm}$. The magnetic moment is reduced due to increasing charge transfer. The projected DOS of the La atom in Fig. 6 reveals the details of electronic state changes. The external field raises the $6 s$ and $5 d$ orbitals for the majority spin, but lowers the $5 d$ orbital for the minority spin. Although the $6 s$ orbital for the majority spin is occupied in the absence of E-field, it moves up with the applied field and loses its occupancy. In the $5 d$ orbital, majority spin occupancy decreases while minority spin occupancy increases with E-field. In the presence of applied uniform E-field, the induced charge density by the impurity charge retains the pattern which is inversely proportional to the cube of the distance from the impurity. If the E-field is in the opposite direction, the spin magnetic moment of La increases and the amount of donated charge decreases. Our results indicate that charging 

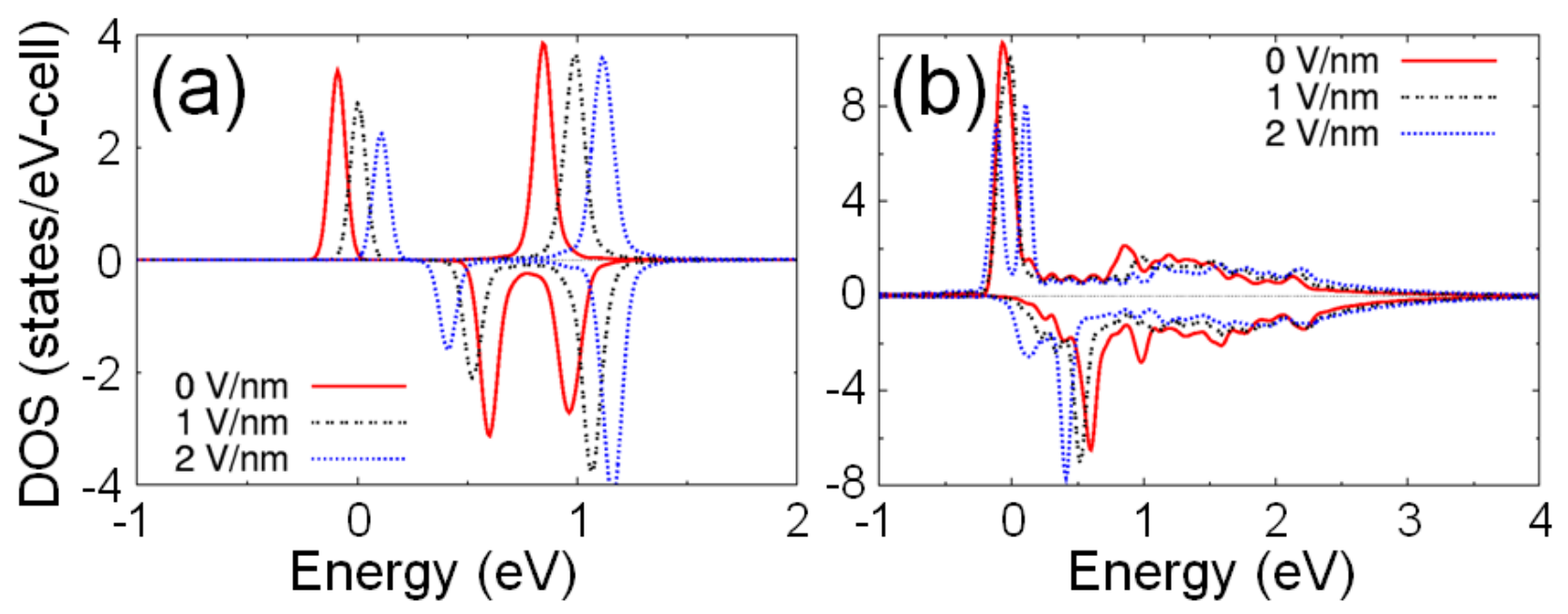

FIG. 6: Orbital-resolved DOS only for La (a) La $6 s$ orbital and (b) La $5 d$ orbital in the La-adsorbed graphene with the increased external field.

and spin magnetic moments could be tailored by adjusting applied E-fields. Therefore, we believe that these findings may shed light on the possibility of electrically tunable (controllable) nano-sized data storage devices.

In summary, we studied the geometric, electronic, and magnetic properties of large-area monolayer graphene with a metal adatom using first-principles calculations. In the two-dimensional STS data, we found that the differences in the band structure modification of the $\mathrm{Cs}$ and $\mathrm{Ba}$ (or La) adatoms originate from the effects of orbital hybridization as well as electron transfer. Interestingly, the total DOS is almost symmetrical around the Dirac point, which implies that far-region graphene states compensate for the asymmetry near the impurity atom. Although the slopes of the LDOS are asymmetrical around the Dirac point because of charge doping and orbital hybridization, the Dirac point is robust. By applying uniform electric field, we showed that field-dependent ionization and changes in spin magnetic moments for metal adsorbates can occur.

This work was supported by the National Research Foundation of Korea through the ARP (R17-2008-033-01000-0) (J.Y.) and the Basic Science Research Program through the NRF of Korea funded by the Ministry of Education, Science and Technology (No. 2010-0007805) (G.K.).

TABLE II: The position (height), charge transfer, and atomic magnetic moment of La atom adsorbed on large unit-cell graphene with an external electric field. Total magnetic moment change of the system also appears.

\begin{tabular}{ccccc}
\hline \hline $\begin{array}{c}\text { field strength } \\
(\mathrm{V} / \mathrm{nm})\end{array}$ & $\begin{array}{c}\text { height } \\
(\AA)\end{array}$ & $\begin{array}{c}\text { charge } \\
(\mathrm{e})\end{array}$ & $\begin{array}{c}\text { atomic moment } \\
\left(\mu_{\mathrm{B}}\right)\end{array}$ & $\begin{array}{c}\text { total moment } \\
\left(\mu_{\mathrm{B}}\right)\end{array}$ \\
\hline 0 & 2.38 & 1.27 & 1.27 & 1.86 \\
1 & 2.27 & 1.43 & 1.04 & 1.77 \\
2 & 2.18 & 1.58 & 0.75 & 1.44 \\
\hline \hline
\end{tabular}


* Electronic mail: gunnkim@khu.ac.kr

[1] J. C. Slonczewski and P. R. Weiss, Phys. Rev. 109, 272 (1958).

[2] K. T. Chan, J. B. Neaton, and M. L. Cohen, Phys. Rev. B 77, 235430 (2008).

[3] B. Uchoa, C.-Y. Lin, and A. H. Castro Neto, Phys. Rev. B , 77, 035420 (2008).

[4] V. A. Rigo, T. B. Martins, A. J. R. da Silva, A. Fazzio, and R. H. Miwa, Phys. Rev. B 79, 075435 (2009).

[5] B. Uchoa, L. Yang, S.-W. Tsai, N. M. R. Peres, and A. H. Castro Neto, Phys. Rev. Lett. 103, 206804 (2009).

[6] A. V. Shytov, M. I. Katsnelson, and L. S. Levitov, Phys. Rev. Lett. 99, 236801 (2007).

[7] M. Caragiu and S. Finberg, J. Phys.: Condens. Matter 17, R995 (2005).

[8] Y. G. Zhou, X. T. Zu, F. Gao, H. Y. Xiao, and H. F. Lv, J. Appl. Phys. 105, 104311 (2009).

[9] http://www.openmx-square.org/

[10] J. P. Perdew, K. Burke, and M. Ernzerhof, Phys. Rev. Lett. 77, 3865(1996).

[11] T. Ozaki and H. Kino, Phys. Rev. B 69, 195113 (2004).

[12] N. Troullier and J. L. Martins, Phys. Rev. B 43, 1993 (1991).

[13] P. E. Blöchl, Phys. Rev. B 41, 5414 (1990).

[14] S. G. Louie, S. Froyen, and M. L. Cohen, Phys. Rev. B 26, 1738 (1982).

[15] J. M. Soler, E. Artacho, J. D. Gale, A. Garcia, J. Junquera, P. Ordejon, and D. Sanchez-Portal, J. Phys.: Condens. Matter 14, 2745 (2002).

[16] S. Meng and S. Gao, J. Chem. Phys. 125, 014708 (2006).

[17] R. D. Diehl and R. McGrath, J. Phys.: Condens. Matter 9, 951 (1997).

[18] Z. Zhu, G. Lu, and F. Wang, J. Phys. Chem. B 109, 7923 (2005).

[19] A. D. Becke and R. M. Dickson, J. Chem. Phys. 89, 2993 (1988).

[20] M. T. Johnson, H. I. Starnberg, and H. P. Hughes, Surf. Sci. 178, 290 (1986).

[21] W. I. Choi, J. Ihm, and G. Kim, Appl. Phys. Lett. 92, 193110 (2008).

[22] V. M. Pereira, J. Nilsson, and A. H. Castro Neto, Phys. Rev. Lett. 99, 166802 (2007).

[23] X. Liu, T. Pichler, M. Knupfer, and J. Fink, Phys. Rev. B 70, 245435 (2004)

[24] M. I. Katsnelson, Phys. Rev. B 74, 201401(R) (2006). 